\title{
EDOS Evolution to Support NASA Future Earth Sciences Missions
}

\author{
Guy R. Cordier \\ Lockheed Martin Mission Systems / GSFC, Greenbelt, MD 20771, USA \\ Bruce McLemore ${ }^{2}$ \\ Honeywell TSI/GSFC, Greenbelt, MD 20771, USA \\ Terri Wood ${ }^{3}$ \\ NASA GSFC, Greenbelt, MD 20771, USA \\ and \\ Chris Wilkinson ${ }^{4}$ \\ NASA GSFC, Greenbelt, MD 2077l, USA
}

\begin{abstract}
This paper presents a ground system architecture to service future NASA decadal missions and in particular, the high rate science data downlinks, by evolving EDOS current infrastructure and upgrading high rate network lines. The paper will also cover EDOS participation to date in formulation and operations concepts for the respective missions to understand the particular mission needs and derived requirements such as data volumes, downlink rates, data encoding, and data latencies. Future decadal requirements such as onboard data recorder management and file protocols drive the need to emulate these requirements within the ground system. The EDOS open system modular architecture is scalable to accommodate additional missions using the current sites antennas and future sites as well and meet the data security requirements and fulfill mission's objectives.
\end{abstract}

\section{Introduction}

$\mathrm{N}$ ASA's Earth Observing System (EOS) Data and Operations System (EDOS) has supported Terra science downlinks at the White Sands Complex (WSC) since December 1999. Previous papers ${ }^{1,2}$ have described this multi-mission high rate system and upgrades to include Aqua, Aura, ICESat, EO-1 and OCO to the mission set. The term EDOS herein refers to the systems and/or the project.

\section{Background}

The EDOS core architecture has remained basically the same through today (Fig. 1). The baseband data are ingested at the stations at downlink rates up to $150 \mathrm{Mbps}$ and the data are rate buffered via high-rate lines to the EDOS's Level Zero Processing Facility (LZPF). There, various level 0 products are generated per standards/formats agreed to by the end-users and distributed to their respective data centers. The data centralization enables the generation of clean continuous data sets, either time-based from all data segments collected at the supporting ground stations or session-based corresponding to a given spacecraft downlink at the site.

\footnotetext{
${ }^{1}$ System Engineer, Sr. Staff, GSFC/ Code 428, Greenbelt, MD 20771

${ }^{2}$ MOMS EDOS Project Manager, GSFC/ Code 428, Greenbelt, MD 20771

${ }^{3}$ EDOS Project Manager, GSFC/ Code 428, Greenbelt, MD 20771

${ }^{4}$ ESMO Deputy Manager, GSFC/ Code 428, Greenbelt, MD 20771
} 


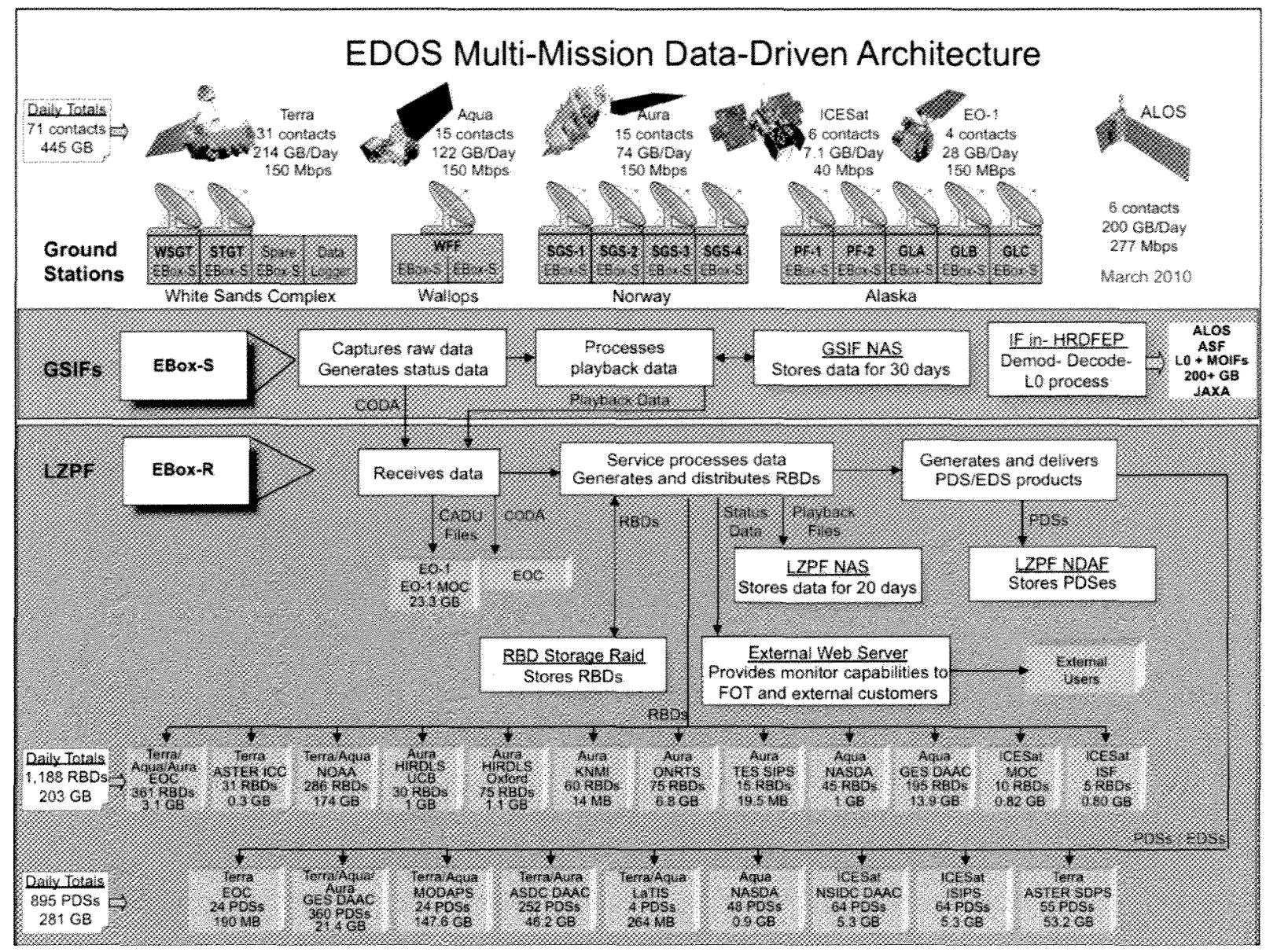

Figure 1. EDOS Distributed Functions and Data Flows

Two key EDOS goals, over the years, have been to reduce mission operations costs and to increase productivity by enabling automation for nominal operation for all system set-up, data capture and processing, and product delivery tasks $\mathrm{s}^{3}$. The system front-end is driven by the acquired signal; the detected spacecraft ID sets the processing configuration according to the mission specific parameters. Technology refresh activities have brought uniformity of hardware platform and operating systems across the sites to simplify equipment/system maintenance activities and system security management. Recently, EDOS deployed a backup LZPF facility at an alternate location for disaster recovery. All missions supported to date conform to the CCSDS standards and use the Reed-Solomon code for communications errors correction.

\section{Architecture Models}

EDOS has two operational architectural models to service the missions' needs: centralized and decentralized. The level 0 functionality performed at the LZPF at GSFC, is also available at a remote site when data are delivered directly to the end-users.

\section{A. Centralized Model}

In the centralized model, all data captured at the remote site is transmitted to the LZPF at GSFC (Fig. 2). The data transfer is accomplished via private high rate NASA networks from the station to GSFC. The LZPF performs level zero processing and generates and delivers all products from GSFC to science customers worldwide. 


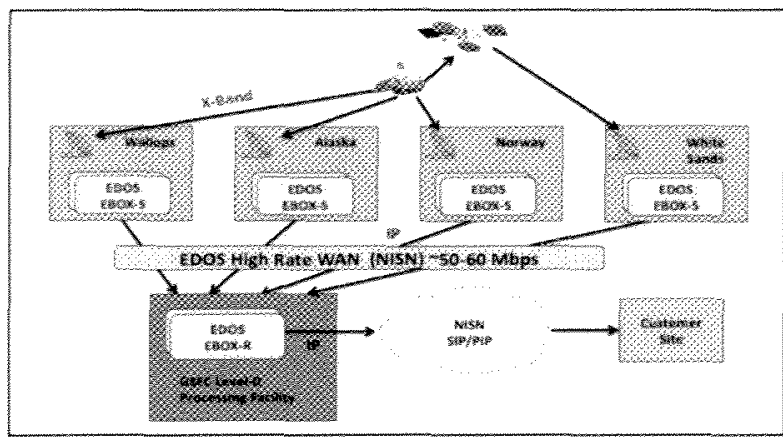

Figure 2. EDOS Centralized Model

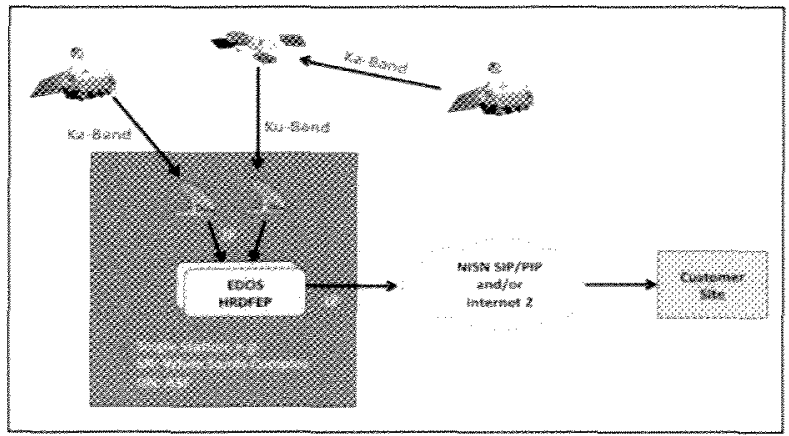

Figure 3. EDOS Remote Site Decentralized Model

\section{B. Single remote site decentralized model}

This EDOS architectural model offers the capability of capturing, processing, and delivering the level zero data directly to the end-users from the ground site, bypassing the GSFC LZPF (Fig. 3). This approach is only applicable if adequate network bandwidth is available and no multiple site processing is required, e.g., Aqua and Aura cannot use this approach. The EDOS modular design permits level zero processing to be performed at the central or at remote sites as required.

\section{Advanced Land Observations Satellite (ALOS) Support at WSC}

In 2008, the EDOS Project was called upon to support a feasibility/demonstration test to process science data downlinked from the Japanese ALOS satellite using the Ka Band, the TDRS Space Network (using the F-10 satellite), and the White Sands Ground Terminal facilities in New Mexico.

EDOS provided a dual systems configuration to capture the baseband signal on one system and the IF signal on the other. The test results demonstrated the benefits of the IF solution. Two identical units have since been installed at WSC to capture the data from the ALOS sensors and are scheduled to begin routine operations in April 2010 (Fig. 4).

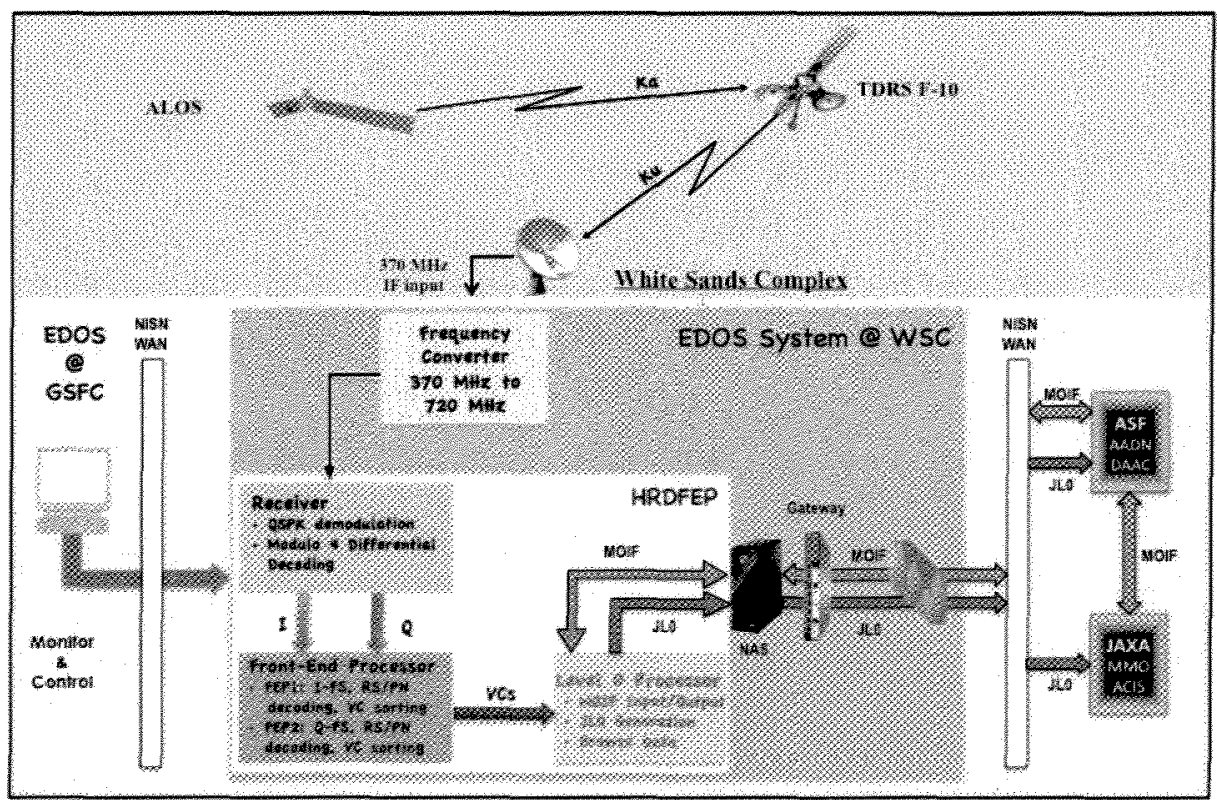

Figure 4. EDOS ALOS System Configuration at WSC 
The systems perform the following functions autonomously: demodulation, decoding, frame synchronization, Reed-Solomon (R-S) error correction, level 0 processing (including browse and quick look image products in realtime), and data distribution directly to the end user's servers at the Alaska Satellite Facility (ASF) and the Japan Aerospace Exploration Agency (JAXA).

\section{New/ Decadal Survey Missions Support}

The Near Earth Network (NEN) Project at NASA/GSFC is planning to upgrade the ground stations resources and equipment capabilities (e.g., antenna, receivers, etc). The NEN project asked EDOS to consider new interfaces to the front-end systems currently deployed at the ground stations and serviced by the existing network of high rate lines.

Working groups meetings and other studies are underway to identify the best configurations to fulfill future Decadal Survey and other Science missions' objectives. Missions compliant to the Consultative Committee for Space Data Systems (CCSDS) data formats standards, having a $300 \mathrm{Mbps}$ downlink rate or below and using the R-S error code can easily be inserted into the mission set and make productive use of existing system capabilities at the station. There are no required changes due to the nature of the serial clock/data interface.

Most new missions however are considering using other error correction codes such as the low-density paritycheck code (LDPC) to achieve better performance or the CCSDS File Delivery Protocol (CFDP) or some variation. Refer to Table 1 for a listing of existing and potential missions with the associated characteristics. In addition, the planned downlink rates for several missions may exceed $1 \mathrm{Gbps}$ and the daily data ingest may be on the order of Terabytes.

Table 1. Mission Characteristics

\begin{tabular}{|c|c|c|c|c|c|}
\hline 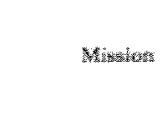 & 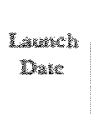 & 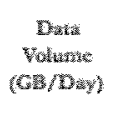 & 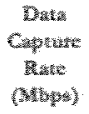 & 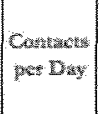 & 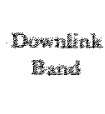 \\
\hline 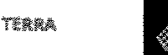 & 199.9 & 25 & 158 & 35 & $\begin{array}{l}\text { TDass } \\
\text { tarbant }\end{array}$ \\
\hline nowa & 2002 & $2 x$ & 150 & 15 & Ftatates \\
\hline $6 x^{2}$ & 2003 & $\pi+2$ & 10 & 5 & whate \\
\hline Astas & 2004 & 74 & 1st & 15 & whane \\
\hline 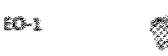 & 2604 & 2 & $1 \$$ & 4 & wbexte \\
\hline ans & 2606 & 24 & $m ?$ & 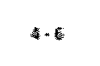 & 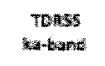 \\
\hline Uxis & 20 & 索 & is & 13 & mbatax \\
\hline 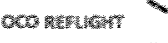 & 2013 & the & 158 & 4 & ebuger \\
\hline 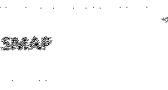 & $\frac{\text { May }}{2013}$ & 138 & Its & 14 & x-kwast: \\
\hline 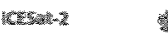 & 2015 & 8 & 40 & 7 & atowate \\
\hline 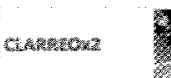 & 2016 & $3.5 \times 2$ & 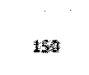 & $5 \times 2$ & oftat \\
\hline 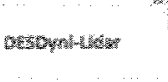 & 2017 & 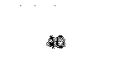 & 130 & $2: 3$ & -bande \\
\hline 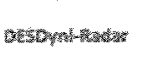 & 2018 & 1000 & 100 & 45 & Tanss \\
\hline Swot & 2020 & 0 & 1000 & 列 & k-tante \\
\hline 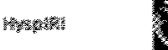 & 3000 & 3000 & 1000 & 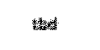 & sa-band \\
\hline
\end{tabular}


The distribution of the functions implemented at the ground stations across the NEN and Space Network (SN), and EDOS resources, respectively has not been settled at this time. There are several factors to take into consideration. EDOS has to evolve its systems to accommodate any new interfaces as it continues to satisfy the current missions' requirements and interfaces. Also, the bandwidth of the high rate lines will need to be resized and upgraded to accommodate the increasing data volumes and the desired latencies for the science data products (see section IV-B).

The EDOS front-end processor, referred to as EBox-S, performs the functions as pictured in Fig. 1. The standard interface at all ground sites accepts a serial bit stream of Channel Access Data Units (CADU) as ECL clock and data in NRZ-L format (except at WSC for the ALOS mission which uses an IF interface). When the NEN hardware/receiver performs the demodulation and decoding with error code corrections, the EBox-S analyzes the incoming signal, recognizes the mission ID, sets up all processing tasks (per stored mission specific parameters), and distributes the data to the end-users - all in an automated/data-driven mode of operation. This method accommodates both existing and new missions with minimal configuration changes. Alternatively, an IP interface could be implemented but would require changes to the associated logic control of the current front-end system.

The EBox-S will have to be upgraded to service the incoming data rate for missions with 1 Gbps downlink rates. The ALOS support at WSC demonstrated the benefits of an IF input into an integrated solution which can be implemented within the existing EBox-S at the ground sites. For example, two additional cards can be added to the existing front-end system in order EDOS to support the SMAP mission and other high-rate missions. The first card will perform the IF signal demodulation; the second card, an updated front-end processor, will perform the decoding and frame processing functions. This front-end processor card would have the necessary FPGA resources to perform the decoding intensive and iterative computing operations associated with all codes under consideration and be capable to ingest data at rates of 1 Gbps and above.

The benefit of such approach, not to mention the modest cost of the modification, enables efficient troubleshooting of issues by EDOS engineering staff who have experience with the interfaces at the ground sites and the end-users facilities. This experience is helpful to pinpoint the source of problems and to implement corrective actions in a timely manner and also for system maintenance and mission end-to-end early testing phase.

As systems engineers for new missions formulate and define the design of on-board data formatting, management and communications subsystems, EDOS expects that there will be new requirements levied on the ground data processing systems, which EDOS can incorporate into its modular design. The EDOS flexibility was amply demonstrated with ALOS where a solution was deployed in a couple of months.

\section{Product Latencies, Network Bandwidth, CFDP, and SLE}

\section{A. Quality of Service (QoS)}

The two EDOS architectures described above (centralized and decentralized) offer multiple means of achieving desired latencies to various end-users with distinct latency requirements. EDOS services include the capability of delivering (1) real-time data streams in under 5 seconds (initially deployed for the Orbiting Carbon Observatory) (2) near real-time "rate-buffered" data in less than 2 hours, and (3) non-real-time session-based or time-based products within 24 hours. For very high rate data mission expected in the Decadal Survey era, the de facto latency is implied by the rate and volume of incoming data and the fact that EDOS must be able to keep up with the daily expected volume with margin for re-transmissions or network outages, if necessary. Managing the various delivery requirements for multiple missions competing for limited available network bandwidth requires the use of QoS capability to assign priorities to data transferred out of the EDOS remote sites. Currently, the WAN bandwidth is approximately $1 / 3$ of the downlink rate for EOS missions, requiring data to be queued and transmitted as bandwidth is available. EDOS deployed the QoS capability at all sites in 2008 to achieve efficient use of NISN WAN resources with excellent results (Fig. 5). Priorities are assigned using TCP port numbers which in turn map to various priority classes to insure efficient use of the network resources. 


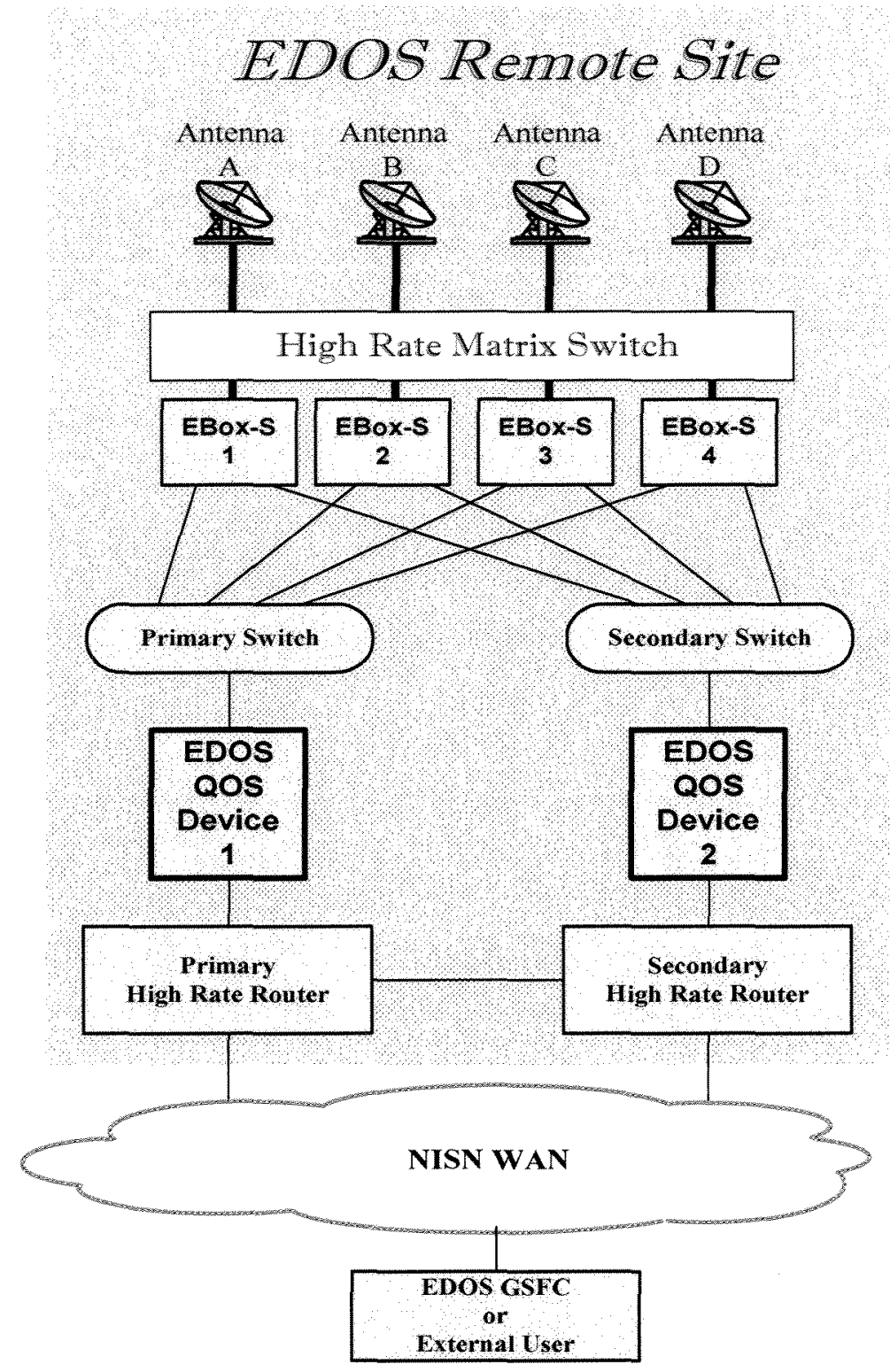

Figure 5. EDOS High-Rate QoS Architecture

\section{B. Network Bandwidth}

Three of the Decadal Survey missions present unprecedented challenges for high-rate data capture, level-zero processing and distribution of data from the remote sites: SWOT, HyspIRI, and DESdynI-Radar. See Table 1 for downlink rates and daily data volumes. Based on expected station utilization and loading studies, estimates were produced for network bandwidth requirements (Fig. 6), based on the addition of Ka-band antennas at Wallops, Svalbard, Alaska, and White Sands. The two EDOS architectural models described above will both be necessary to support these 3 missions. The centralized model can be used to support SWOT and HyspIRI missions with additional WAN bandwidth (Fig. 6), and the remote site de-centralized model (ALOS model) can be used to support DESdynI-Radar at White Sands with additional bandwidth added to connect White Sands to the NISN backbone (Fig. 6). All of these missions will require additional network resources at each site. The de-centralized model is particularly applicable for DESdynI-Radar due to the high data volume (approximately 5 Tbytes/day) being transferred directly to the Jet Propulsion Laboratory (JPL) via the commercial NISN backbone. The main reasons 
supporting the feasibility of this design are: (1) the NISN backbone has a presence at the White Sands location providing high-rate network access to JPL; (2) this is science data and does not require the security of a mission network; (3) expanding the NISN EBnet WAN to the required bandwidth would be extremely expensive; and (4) the data would have to travel to GSFC and then be returned to JPL anyway. The modularity of EDOS components allow the required level-zero processing functions to reside at the remote site as well as at GSFC's LZPF. Additionally, EDOS components and associated network interfaces are already located in or near all the planned remote site facilities.

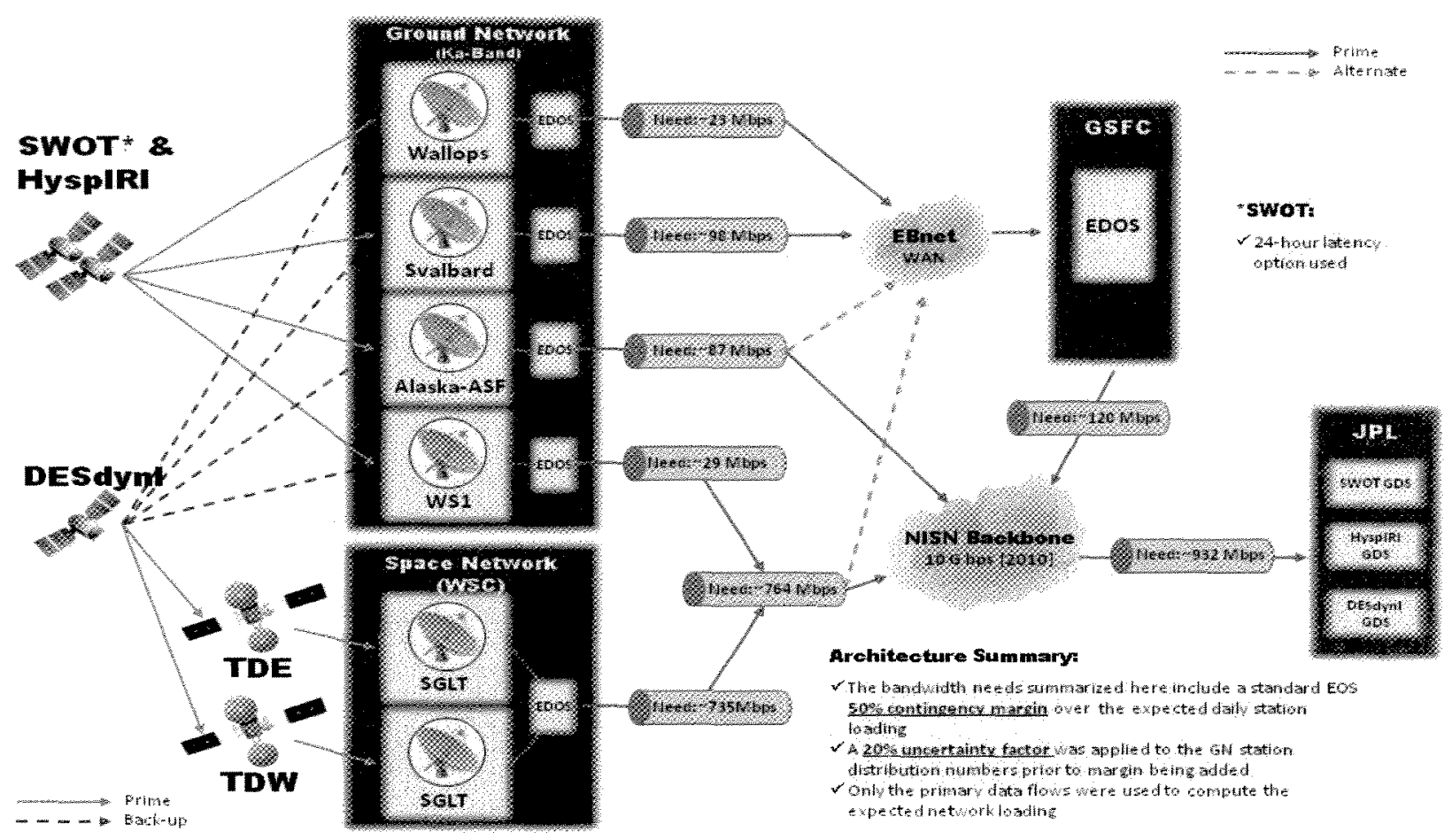

Figure 6. High-Rate WAN Architecture (EDOS) For Decadal Survey Missions

\section{CCSDS File Delivery Protocol (CFDP)}

In support of the Decadal Survey missions, EDOS plans to implement both class 1 CFDP (i.e., send data once and get no feedback from the receiver) and class 2 CFDP (send the data once, then use feedback to resend any missing data) within the next 2 years (Fig. 7) using the CFDP engine developed at GSFC by Tim Ray (GSFC Code 583). EDOS presents a challenge to single-site implementations of CFDP (e.g. the Solar Dynamics Observatory, Lunar Reconnaissance Observatory) with four remote stations (and more on the horizon). Downlinks at one station may require a retransmission at the next downlink to another station. EDOS will support CFDP with the centralized model providing centralized CFDP processing and file distribution from the LZPF. The centralized model of EDOS can support the lower rate missions and/or latency tolerant missions with centralized CFDP processing providing accounting reports, or metadata, to the project Control Center, as well as a generic command interface for class 2 CFDB. As discussed above, the high rate Decadal Survey missions will have limited delivery bandwidth across the WAN to the LZPF, therefore the increased latency for retransmission can be eliminated using the remote site decentralized model for these missions and implementing a CFDP accounting process at the remote sites. Stations reports (e.g., of gaps, metadata) could be sent to the project Control Center by capturing on one pass and then retransmitting on the next pass, if necessary. Reports of successful file creation provide verification that the received file is complete to the MOC. This approach is under evaluation and not necessarily final. 


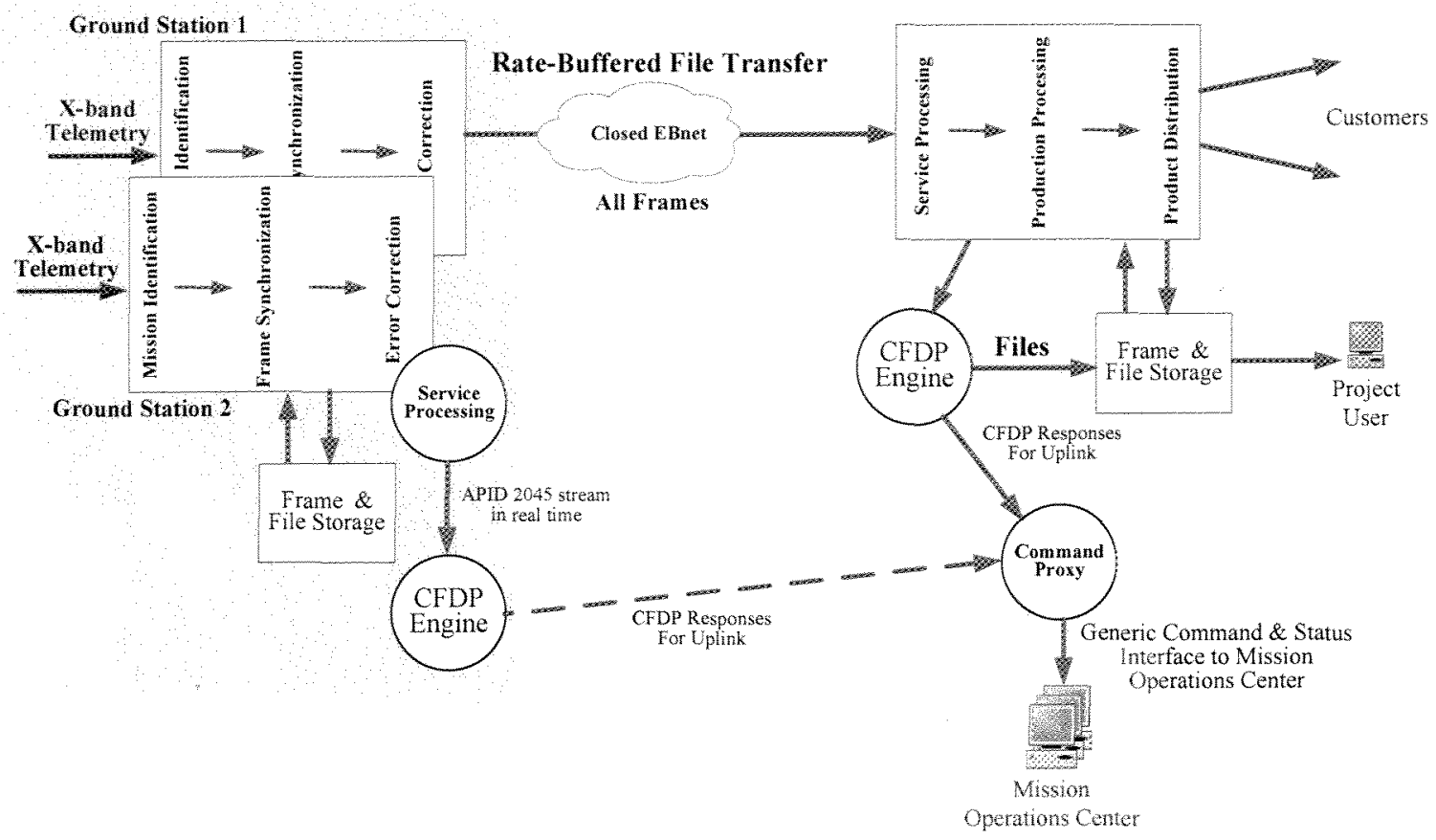

Figure 7. EDOS CFDP Architecture

\section{Space Link Extension (SLE)}

EDOS plans to add Space Link Extension (SLE) capability via compliant tcp socket interfaces to the EBox-S, to enable the role of a SLE provider for future missions (Fig. 8). We anticipate that EDOS will provide Return All frames (RAF) or Return Channel Frames (RCF) services to mission science users or control centers. For OCO, for example, EDOS implemented the capability of extracting the VC0 and VC1 (housekeeping data) in real-time from the X-band downlink and streaming the frames to the MOC in less than 1 second.

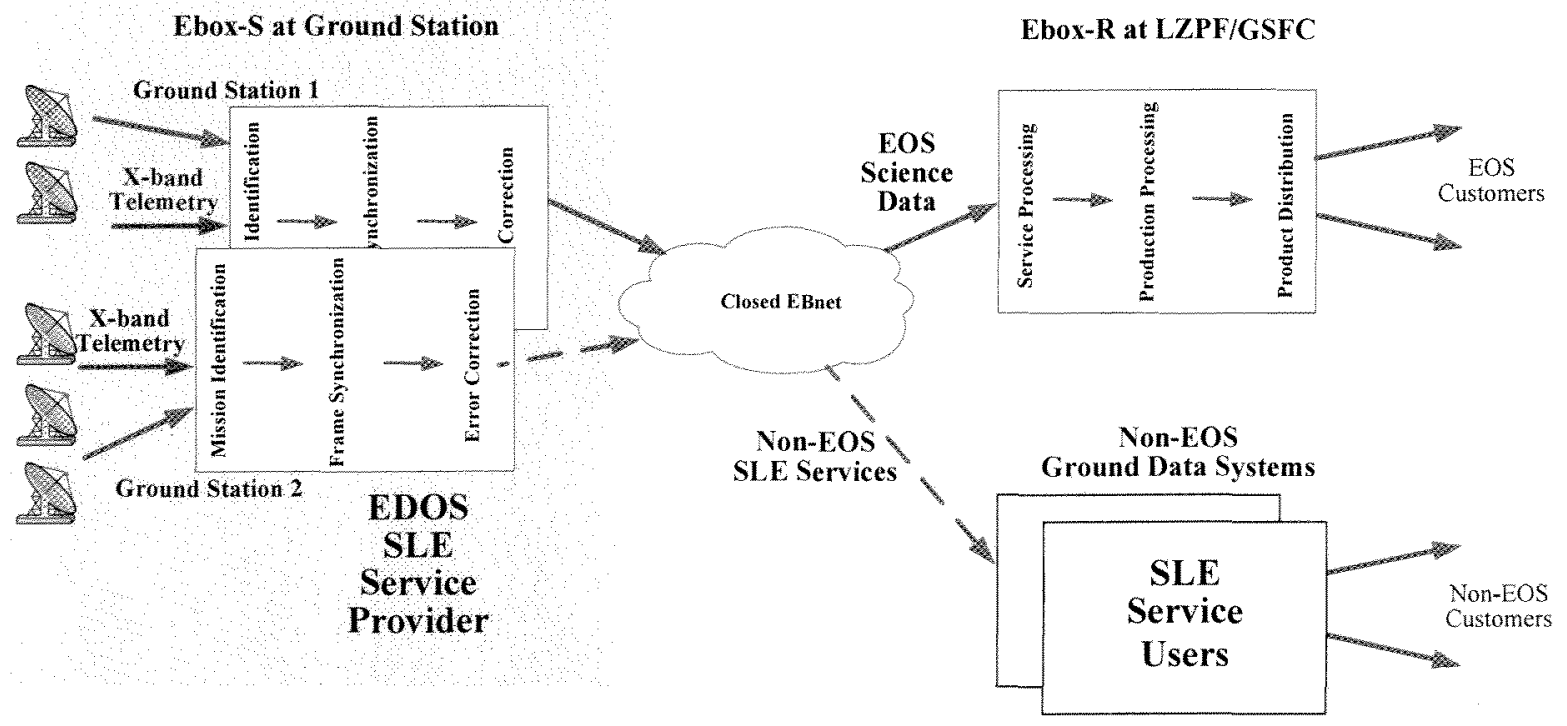

Figure 8. EDOS SLE Architecture 


\section{Other near-term developments}

Continuous improvements of the operational systems are always under consideration as supporting technologies advance, e.g., data storage, processor speed, FPGA resources as requirements dictate. EDOS has a sustaining engineering team with a strong operational experience dedicated to the task. This group partners with the Kongsberg Spacetec systems engineering group to incorporate new functionalities/capabilities into the existing systems per new mission requirements.

The authors have previously reported on EDOS capabilities to provide via web browser various views into the data quality in real-time during the downlinks at the stations. An automated process to provide data continuity reports in near real-time to the mission operations control centers is planned for Decadal Survey missions support. The automation applied to monitoring systems functions, products generation and products distribution will enable the system to report exceptions to operations personnel and perform otherwise manual data accounting tasks.

When automated mode of operation for Aqua and Aura spacecrafts is enabled, EDOS will reconstruct instrument packets from successive frames downlinked in different contacts. With manual operations, this is taken care of by the downlink process of overlap data.

EDOS external product servers are about to be deployed enabling end-users to retrieve past products that, for one reason or another, are missing.

Also, EDOS product latencies are being reduced by optimizing the data content associated with the data ratebuffered from the stations to the LZPF, e.g. elimination of R-S code, enabling data compression.

\section{Applicable recent lessons learned}

The data-driven concept for the EDOS systems has enhanced overall reliability and automation and enabled lights-dim operations. In a real life example, during the unusually severe snow storm of February 2010, support of the ALOS tests was on-going and all passes were captured and the data were processed and distributed in a lightsout mode of operation for several days.

The availability of the raw telemetry data and the practical means, tools, and skills to analyze it have proven to be key when troubleshooting anomalies in the raw data bit streams.

\section{Acknowledgments}

The authors express their appreciation to the NASA GSFC civil servants and contractor personnel who contributed to the EDOS project over the years. A special mention of appreciation is extended to the Mission Operations and Mission Services (MOMS) EDOS support team at GSFC and to the Kongsberg Spacetec team of Tromso, Norway for their professional services and technical support.

The contractor support for this paper was provided under the Honeywell Technology Solutions Inc. (HTSI) MOMS contract \# NNG04DA01C with NASA.

\section{References}

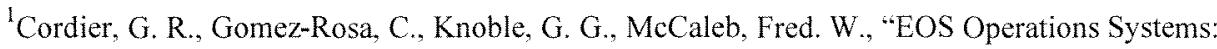

Planned and Proposed Changes to Reduce Operations Costs," Proceedings of the 6th International Symposium on Reducing the Costs of Spacecraft Ground Systems and Operations, SP-601, ESA, 2005

${ }^{2}$ Cordier, G.R., Gomez-Rosa, C., Knoble, G. G., McCaleb, Fred. W..," Autonomous multi-missions, multi-sites X-band data capture systems, "SpaceOps 2006 Conference, Rome, Italy

3Cordier, G.R., Gomez-Rosa, C., McLemore, B., "EOS Operations Systems: EDOS Implemented changes to reduce Operations Costs" Proceedings of the $7^{\text {ih }}$ International Symposium on Reducing the Costs of Spacecraft Ground Systems and Operations, SP-648, ESA, 2007

4Cordier, G. R., Wilkinson, C., C., McLemore, B., "Web monitoring of EOS front-end ground operations, science downlinks and level 0 processing, "SpaceOps 2008 Conference, Heidelberg, Germany 